\title{
Warm dust and gas of massive young stellar objects revealed by Herschel PACS spectroscopy
}

\author{
Woojin Kwon ${ }^{1,2}$, Floris F. S. van der Tak ${ }^{2,3}$, Agata Karska ${ }^{4,5,6}$, \\ Gregory J. Herczeg ${ }^{7}$, Luis Chavarría ${ }^{8}$, Fabrice Herpin ${ }^{9,10}$, Friedrich \\ Wyrowski $^{11}$, Jonathan Braine ${ }^{9,10}$, and Ewine F. van Dishoeck ${ }^{5,6}$ \\ ${ }^{1}$ Korea Astronomy and Space Science Institute, 776 Daedeokdae-ro, Yuseong-gu, Daejeon \\ 34055, Republic of Korea, email: wkwon@kasi.re.kr \\ ${ }^{2}$ SRON Netherlands Institute for Space Research, Groningen, The Netherlands \\ ${ }^{3}$ Kapteyn Astronomical Institute, University of Groningen, Groningen, The Netherlands \\ ${ }_{4}^{4}$ Astronomical Observatory, Adam Mickiewicz University, Poznan, Poland \\ ${ }^{5}$ Max-Planck Institut für Extraterrestrische Physik (MPE), Garching, Germany \\ ${ }^{6}$ Leiden Observatory, Leiden University, Leiden, The Netherlands \\ ${ }^{7}$ Kavli Institute for Astronomy and Astrophysics, Peking University, Beijing, PR China \\ ${ }^{8}$ Universidad de Chile - CONICYT, Santiago, Chile \\ ${ }^{9}$ Université de Bordeaux, Floirac Cedex, France \\ ${ }^{10}$ CNRS, LAB, Floirac Cedex, France \\ ${ }^{11}$ Max-Planck Institut für Radioastronomie, Bonn, Germany
}

\begin{abstract}
We present results of Herschel PACS imaging spectroscopy data toward ten massive young stellar objects taken as part of the WISH project. Our sample consists of four high mass protostellar objects (HMPOs), two hot molecular cores (HMCs), and four ultracompact HII regions (UCHIIs), and the spectra cover a broad range of wavelengths (55 to $210 \mu \mathrm{m}$ ) imaged over an $\sim 50^{\prime \prime}$ field with $5 \times 5$ spaxels. By fitting the continua utilizing a modified black-body formula we estimate mass-weighted dust temperature and column density distributions of warm dust and find that UCHII regions are hottest and HMCs are most deeply embedded. We also estimate rotational temperature and column density distributions of warm $\mathrm{CO}$ gas using the rotational diagram analysis, which are comparable over targets in contrast to continuum results. By comparing high J CO line fluxes to the RATRAN estimates of centrally heated envelope models, we find that majority of warm $\mathrm{CO}$ originates from bipolar outflow shocks.
\end{abstract}

Keywords. astrochemistry, stars: formation, stars: pre-main-sequence, HII regions

\section{Introduction}

Warm dust collision is the main heating mechanism of gas in quiescent molecular clouds (Doty \& Neufeld 1997). However, when stars form, various heating mechanisms are added: energetic bipolar outflows and strong UV photons. We investigate physical properties of dust and gas (temperature and column density distributions) around massive young stellar objects and discuss whether the properties are explained by centrally heated envelope models (van der Tak et al. 2013).

Ten massive young stellar objects, which consist of four high mass protostellar objects (HMPOs: DR21(OH), W3 IRS5, W33A, AFGL 2591), two hot molecular cores (HMCs: G327-0.6, NGC 6334-I), and four ultra compact HII regions (UCHIIs: G5.89-0.39, G34.26+0.15, NGC 7538-IRS1, W51N-e1), have been observed by the Photodetector Array Camera and Spectrometer (PACS, Poglitsch et al.2010) of the Herschel Space Observatory (hereafter Herschel), as part of the Herschel guaranteed time key program, Water in star-forming regions with Herschel (WISH, van Dishoeck et al. 2011). The PACS imaging spectroscopy data offer broad spectra of 55 to $210 \mu \mathrm{m}$ in $5 \times 5$ spatial pixels (hereafter spaxels, $9.4^{\prime \prime} \times 9.4^{\prime \prime}$ each), which include dust 
continua and various atomic and molecular lines. The central spaxel data of our dataset have been employed in Karska et al. (2014a) for studying molecular cooling in star formation.

\section{Warm dust}

First, we estimate deconvolved sizes of dust continuum at $170 \mu \mathrm{m}$ using the 2D Gaussian function and find that they are smaller than those at $450 \mu \mathrm{m}$. This implies central heating: cold dust grains are farther distributed.

In addition, we fit individual continua of 25 spaxels over the PACS wavelengths using the modified blackbody radiation formula for constraining mass-weighted temperature and column density distributions. The mass-weighted dust temperatures and column densities are broadly ranged: e.g., $T_{d}=35-60 \mathrm{~K}$ and $N=0.5-4.0 \mathrm{~g} \mathrm{~cm}^{-2}$ at the center. However, we find that UCHIIs have the highest mass-weighted dust temperatures and HMCs have the highest column densities implying the most deeply embedded state.

We also compare the temperature and column density distributions with centrally heated envelope models (van der Tak et al. 2013). Such passive envelope models well explain the Herschel PACS observations, but the outer regions appear to be warmer than the models, which suggests an external heating.

\section{Warm CO}

We obtain $\mathrm{CO}$ rotation temperatures and column densities using the rotational diagram analysis based on CO ladders of $J_{u p} \geqslant 14$. Rotation temperatures of CO $\left(T_{\text {rot }}=150-350 \mathrm{~K}\right.$ at the center) are much higher than the mass-weighted dust temperatures. In addition, they are comparable over radius and objects unlike dust temperatures. Column densities of $\mathrm{CO}$ $\left(N_{C O}=0.1-1 \times 10^{16} \mathrm{~cm}^{-2}\right)$ do not indicate a significant variation over objects either, differently from dust continuum estimates. The discrepancy suggests that the components traced by warm $\mathrm{CO}$ differ from what continuum tracks.

In addition, we calculate $\mathrm{CO}$ fluxes of centrally heated envelope models using RATRAN (Hogerheijde \& van der Tak 2000) and compare them with data. The comparison shows that majority of warm $\mathrm{CO}$ cannot be explained by envelope heating. The fraction of warm $\mathrm{CO}$ understood by envelope heating is about $20 \%$ at the central regions of all the three type objects (HMPOs, HMCs, UCHIIs). When the whole PACS $5 \times 5$ spaxel area is taken into account, the fraction is only about $10 \%$ or less. This indicates that the main heating mechanism of warm CO is more extended than an envelope. Outflow activities like UV photons through bipolar outflow cavities and outflow shocks are thought to be the main excitation mechanisms of warm $\mathrm{CO}$ (Karska et al. 2014b).

\section{References}

Doty, S. D. \& Neufeld, D. A. 1997, ApJ, 489, 122

Hogerheijde, M. R. \& van der Tak, F. F. S. 2000, A\&A, 362, 697

Karska, A., Herpin, F., Bruderer, S., et al. 2014a, A\&A, 562, 45

Karska, A., Kristensen, L. E., van Dishoeck, E. F., et al. 2014b, A\&A, 572, A9

Poglitsch, A., Waelkens, C., Geis, N., et al. 2010, A\&A, 518, L2

van der Tak, F. F. S., Chavarría, L., Herpin, F., et al. 2013, A\&A, 554, A83

van Dishoeck, E. F., Kristensen, L. E., Benz, A. O., et al. 2011, PASP, 123, 138 